\title{
EXPLORACIÓN DE LAS COMPETENCIAS DE LOS ORIENTADORES PROFESIONALES EN CONTEXTOS SOCIOLABORALES
}

\section{COUNSELLOR PROFESSIONAL COMPETENCIES IN SOCIAL AND LABOUR ENVIRONMENTS}

\author{
Luis Sobrado*, Ana Couce**, Raquel Rial*** \\ Universidad de Santiago de Compostela
}

\section{RESUMEN}

En este artículo se plantea la competencia profesional del Orientador Laboral entendida como el dominio de conocimientos, actitudes, habilidades y su saber hacer como consecuencia de la formación adquirida.

El estudio que se presenta es el resultado de una investigación efectuada con un colectivo de 81 Orientadores Profesionales que ejercen en la Comunidad Autónoma de Galicia y que trata de los objetivos siguientes:

$1^{\circ}$. Describir el perfil personal y profesional de los Orientadores Laborales.

$2^{\circ}$. Diagnosticar las competencias orientadoras que realizan en su práctica profesional, las que deberían efectuar y la preparación en ellas.

$3^{\circ}$. Explorar las dimensiones correspondientes a las competencias orientadoras.

Como metodología investigadora se aplicó la técnica Delphi con un grupo de doce orientadores expertos y un cuestionario de competencias a valorar por el colectivo de 81 profesionales de la Orientación Laboral.

Como conclusiones principales del trabajo destacan las competencias profesionales de Asesoramiento, Orientación y Diagnóstico en el grupo estudiado.

El perfil predominante del Orientador descrito corresponde a una persona joven, de sexo femenino, con reducida experiencia profesional en general y orientadora específicamente, titulación académica de Licenciatura en Pedagogía, acceso laboral mediante contratación temporal y que ejerce en una Oficina Pública de Empleo.

Descriptores: Competencias; Diagnóstico; Profesional; Orientación; Asesoramiento; Orientador.

* Luis Sobrado Fernández es catedrático de Métodos y Técnicas de Investigación Educativa en Ciencias del Comportamiento, y de la Educación, en la Universidad de Santiago de Compostela. Investiga sobre la Orientación Educativa y Profesional con numerosas publicaciones. 
Curriculum Profesional: Catedrático de Universidad de Diagnóstico y Orientación, Departamento de Métodos y Técnicas de Investigación de la Universidad de Santiago de Compostela (Luis Sobrado Fernández).

Becarias de investigación (predoctorales) del Departamento y Universidad referenciados (Ana Couce Santalla y Raquel Rial Sánchez).

Líneas de Investigación: Diagnóstico de necesidades y competencias orientadoras; Evaluación de Servicios y Programas de Orientación; Desarrollo Profesional de Orientadores; Orientación y acción tutorial; Inserción Profesional.

Fecha de finalización del trabajo: Septiembre del 2002.

\begin{abstract}
In this article the profesional competence of the Laboral Counsellor is debated understood as the power of knowledge, attitudes, skills and knowhow as a consecuence of the achieved training.

The study that is shown is the result of a investigation made with a team of 81 Profesional Counsellors that practise in the Galician Autonomous Community and that is about the following aims:

$1^{\circ}$. To describe the personal and professional profile of the Laboral Counsellors.

$2^{\circ}$. To diagnose the counselling competences that make in their professional practice, the ones that they should make and the preparation in them.

$3^{\circ}$. To explore the dimensions that are related to guidance competences.
\end{abstract}

As a $n$ investigating methodology the Delphi technique was worked with a group of twelve expert Counsellors and a questionnaire of competences to be valued by the team of 81 Professionals in the Laboral Guidance.

As the main conclusions of the work the professional competences of Advising, Guidance and Diagnosis in the studied group.

The main profile of the Counsellor that is described corresponds to a young person, female, with little professional experience in general and specifically counselling, with an academic degree in Pedagogy, labour access through temporal hiring and that practise in an Employment Public Office.

Descriptors: Competences; Diagnosis; Professional; Guidance; Advising; Counsellor.

Professional Curriculum: professor of Diagnosis and Guidance, Department of Methods and Investigation Techniques of the University of Santiago of Compostela.

Investigation plan: Diagnosis of the necessities and professional competences; Evaluation of services and Guidance Programmes; Professional development of

Counsellors; Guidance and tutorial action; Professional Insertion.

Date of the end of work: September 2002.

\title{
Introducción
}

Entre las distintas acepciones del término competencia en el contexto profesional se halla la vinculada con la formación, refiriéndose al nivel de capacitación en conocimientos, actitudes, habilidades, del saber hacer de una persona como consecuencia de su preparación adquirida.

En la situación de los profesionales de la Orientación en la que nos encontramos la competencia se refiere a los saberes, capacidades y habilidades que son necesarias desarrollar mediante la formación inicial y continua (Eraut, 1997). 
Además cabe considerar también aquí la competencia como cualificación, relacionada básicamente con la preparación necesaria para poseer la capacidad profesional que se desea. De este modo la competencia es el resultado del proceso de cualificación que posibilita «ser capaz de orientar» y estar preparado para «orientar».

La competencia profesional del Orientador se refiere pues a los roles, funciones y tareas a desarrollar adecuadamente por éste en su puesto de trabajo y que son consecuencia de un proceso de formación y de cualificación profesional (Sanz, 1999).

Las competencias profesionales las podemos clasificar en genéricas o transversales y específicas (Levy Leboyer, 1997). Las genéricas o transversales poseen mayor grado de transferencia de unas actividades profesionales a otras, mientras que las específicas son propias de una profesión concreta. Esta cuestión posee implicaciones en la formación, pues en el caso de las competencias genéricas parece existir mayor conexión con su multifuncionalidad o polivalencia y en este sentido la preparación de base está más en consonancia con las competencias generales (Mertens, 1998).

Dentro de las capacidades genéricas es preciso citar las cognoscitivas o aptitudes, los conocimientos adquiridos tanto teóricos como experienciales, las tendencias del comportamiento o actitudes, los valores y rasgos de la personalidad y las habilidades o destrezas (Mazariegos y otras, 1999).

\section{Diagnóstico de las competencias genéricas}

La necesidad de adecuar la formación de los profesionales en general y de los Orientadores en particular a las demandas reales y cambiantes de la sociedad y del ámbito laboral, ha puesto sobre el tapete la atención sobre nuevas dimensiones de la profesionalidad como son la introducción de habilidades en relación con los conocimientos básicos, las capacidades de relación, de adaptación, de «aprender a aprender», etc. (ISFOL,1992). A esta modalidad de competencias se las ha denominado genéricas, «competencias claves» o «competencias transversales», en el sentido de su grado de generalidad, polivalencia y multifuncionalidad y que son transferibles y aplicables en diferentes contextos, profesiones, tareas, etc., (Anta, 1998).

En el ámbito de competencias profesionales existen ciertas diferencias entre los sistemas británico, alemán y francés que conviene considerar para una mayor ilustración al respecto.

En Gran Bretaña (RVQ,1986) las competencias genéricas se las denomina «Key skills» y garantizan su transferencia y aplicación a diversos contextos. Sus unidades de formación estándar son la comunicación, informática, trabajo en equipo y mejora del aprendizaje y su puesta en práctica.

El sistema alemán posee las Shlussel-Qualificationen, referentes a conocimientos y habilidades para desarrollar funciones y afrontar los cambios en la vida profesional. Es un modelo que ha desarrollado el aprendizaje a través de la intervención directa en las organizaciones sociolaborales (Bunk, 1994).

Las competencias se hallan integradas en el sistema dual mediante un modelo pedagógico basado en metodologías activas que pueden evaluarse de una forma integrada. Considera cuatro tipos de competencias que son las técnicas (conocimientos, nociones, saberes, apti- 
tudes), metodológicas (procedimientos, habilidades), sociales (actitudes, sentimientos sociales) y participativas (modos de organizarse, trabajos en grupo, etc.). La integración de todas estas modalidades genera las denominadas competencias de acción y el acentuar éstas facilita sus experimentación y evaluación en el lugar de trabajo.

En el sistema francés (Joras y Ravier, 1993) el objetivo del Balance de Competencias es construir un proyecto personal y profesional a partir del análisis de necesidades y de los recursos laborales y sociales. El proyecto debe estar en relación con la persona y por ello es preciso conocer sus motivaciones, recursos, etc.

El proyecto profesional debe poder realizarse en el mercado de trabajo y por ello se observa lo que existe y cuáles con las competencias necesarias para llevarlo a efecto tanto en el «saber», como en el «saber estar»y en el «saber hacer».

La persona debe hallar un lugar de trabajo para experimentar el proyecto, reflejarlo y ser escuchado. El proceso se efectúa con una serie de metodologías que exigen honestidad, rectitud, responsabilidad, etc., a la persona que decida realizarlo.

El sujeto debe ser capaz de manejarse autónomamente, conocer sus propios recursos, sus limitaciones y que es lo que debe cambiar o mejorar. Deberá ser consciente de sus propios valores y de cómo emplearlos en un lugar de trabajo y proceder con una dinámica constructiva (Ceri, 1996).

En nuestro país la LOGSE trata de los «ejes transversales» y de «la educación de valores» términos próximos a las competencias. La reforma actual del sistema de Formación Profesional enfatiza también las competencias de tal modo que el perfil profesional se define mediante la competencia general que describe sistemáticamente los objetivos nucleares de la profesión.

Las competencias profesionales y las capacidades clave son de tipo personal más asociadas a comportamientos observables en el sujeto y consecuentemente son transversales en el sentido que repercuten en muchos puestos profesionales y son transferibles a nuevas situaciones laborales.

Las «certificaciones de profesionalidad» que se están articulando en el ámbito estatal español sirven para acreditar las competencias profesionales que incluyen el desarrollo de competencias claves como son los saberes para adquirir otros conocimientos, actitudes y habilidades vinculadas con la profesión, etc. (Ministerio de Educación y Cultura, 1998).

Las competencias transversales son un conjunto de capacidades, actitudes y habilidades imprescindibles para llevar a buen término un trabajo profesional. Éstas son transferibles a nuevas situaciones de trabajo.

Como competencias transversales podemos mencionar el autoconocimiento de las propias potencialidades que posee la persona, estar motivado profesionalmente, poseer capacidad de adaptación y autocontrol, saber comunicar en las relaciones, ser capaz de trabajar en equipo, de resolver problemas y de adaptarse al contexto profesional (Blagg y otros, 1993).

Como competencias generales en el ámbito orientador se pueden mencionar la atención a la diversidad social y cultural, la utilización de un código ético de actuación profesional, la comunicación y las relaciones interpersonales, el diseño, realización y evaluación de programas de orientación, la valoración de las necesidades de los orientadores, etc. 
Desde la perspectiva de las competencias genéricas es de interés el estudio de Hiebert (2000a) de la Universidad de Calgary que se refiere dentro del esquema del modelo de competencias a los estándares y directrices del Desarrollo de la Carrera en Canadá. Señala las siguientes áreas de competencias centrales del Orientador: Comportamiento profesional, competencia interpersonal, conocimiento del desarrollo de la carrera y evaluación de necesidades y referencias.

\section{Evaluación de las competencias específicas}

Las competencias de naturaleza específica son más apropiadas en el ámbito de la especialización, y por ello la formación continua estará más en consonancia con ellas. En este sentido las competencias técnicas se corresponden más con las específicas y las metodológicas estarían a caballo entre éstas y las genéricas.

Se entiende la competencia técnica como el dominio como experto de las tareas y contenidos del ámbito de trabajo propio y los conocimientos y habilidades necesarios para ello y por competencia metodológica la aplicación de procedimientos adecuados a las tareas y funciones propias de la profesión concreta que encuentra de una manera autónoma vías de solución y que transfiere adecuadamente las experiencias adquiridas a otras modalidades laborales. Las competencias específicas de orientación hacen referencia al Asesoramiento entendido como ayuda personal y grupal en ámbitos de carácter académico, profesional, familiar, etc.; la orientación para el grupo en problemas de integración; métodos y técnicas de aprendizaje y de estudio; en habilidades de toma de decisiones; actividades de comunicación interpersonal, etc.

Como áreas de especialización dentro del estudio de Hiebert (2000b) sobre el modelo de competencias de los estándares y directrices del Desarrollo de la Carrera en Canadá figuran las siguientes: Evaluación, Facilitación del Aprendizaje, Elaboración de la capacidad de relaciones con la comunidad, Asesoramiento para la carrera, Dirección de información y Recursos y desarrollo del trabajo.

En síntesis en el modelo canadiense de estándares y directrices para el desarrollo de la carrera existen competencias centrales (genéricas) y competencias por áreas de especialización (específicas).

A su vez se dividen en áreas de competencia (seis en las centrales y otras seis específicas con dos más comunes a varias áreas de especialización). Asimismo dentro de cada Área de competencia existen funciones orientadoras y a su vez se desglosan en competencias necesarias de naturaleza más concreta abarcando 89 en total.

Además de las competencias centrales o clave, los Orientadores precisan poseer competencias especializadas que varían en función del servicio que se facilite, del tipo de persona que se está atendiendo, etc.

Las áreas de especialización con sus competencias específicas pueden considerarse como grupos de competencias implicadas para proporcionar los diversos servicios que las personas puedan precisar.

La conexión entre las competencias genéricas (centrales) y las específicas (área de especialización) se considera no jerárquica y poseen igual rango. Ninguna área se considera más 
o menos importante que las demás y todas las competencias son relevantes para facilitar servicios de orientación apropiados.

Respecto al modelo de competencias de Canadá de estándares y directrices para el desarrollo de la carrera, en el estudio efectuado por Hiebert (2000a) las sugerencias efectuadas por los participantes en las consultas de campo sobre su utilización potencial fueron en la idea de que pueden emplearse por los Orientadores como una guía para la autoevaluación, para valorar sus puntos fuertes y débiles y planificar su propio desarrollo profesional.

Los estándares pueden también usarse por los propios supervisores y como guía para desarrollar programas de formación inicial y continua de los profesionales de la Orientación. Asimismo como recurso para valorar su preparación previa lo que podría desembocar en una formación mas consistente y comprensiva.

Por otra parte los estándares pueden ser utilizados por las Asociaciones Profesionales de los Orientadores para fomentar una especialización en la carrera profesional e incluso para autorizar la práctica de su profesión.

De lo expuesto anteriormente se deduce que son necesarios estudios e investigaciones para identificar las competencias profesionales y definir el modelo o estándar que debe adoptar la cualificación profesional de los Orientadores para ser incorporado al Sistema Nacional y al catálogo correspondiente de las profesiones.

Existe una identificación de las competencias de los Orientadores generadas a partir de las investigaciones de Watts y colaboradores (1992), Ballesteros (1996), Sobrado (1997, 2002), Sanz Oro (1999), Hiebert (2000a) y Repetto (2000).

Básicamente nos fundamentaremos en las competencias generales de los Orientadores Escolares y Profesionales de carácter transversal y clave, identificadas y en las dimensiones orientadoras y sus correspondientes competencias específicas. Son fundamentalmente las de Diagnóstico, Orientación Personal y grupal, Orientación y Desarrollo Profesional, Asesoramiento, Gestión de la información y los recursos, Coordinación y consulta, Investigación y Evaluación, Dirección de Servicios y/o Programas Orientadores, Relaciones con la Comunidad e Inserción Profesional.

\section{Análisis de los datos e interpretaciones de los resultados (competencias profesionales de los Orientadores)}

\section{Descripción de los profesionales de la Orientación}

a) Muestra

Está integrada 81 Orientadores Profesionales que ejercen en instituciones laborales.

Dicha muestra abarca aproximadamente el 36\% de la población actual de Orientadores existentes en Galicia integrada por 270 componentes de los Servicios de Orientación Profesional en el ámbito ocupacional que ejercen en la Comunidad Gallega.

Se trata pues de una muestra relativamente amplia de profesionales de la Orientación Laboral. 
El cuestionario de competencias orientadoras fue remitido por correo a todos los Orientadores Laborales que ejercen en la Comunidad Autónoma Gallega. Las principales informaciones personales y profesionales se mencionan seguidamente.

b) Datos personales y profesionales

$\left.1^{\circ}\right)$ Edad.

TABLA 1: Edad.

\begin{tabular}{|c|c|}
\hline $\begin{array}{c}\text { TRAMOS EDAD } \\
(\%)\end{array}$ & NIVELES \\
\cline { 2 - 3 } & Orientación Laboral \\
\hline 1. Menor de 35 años & $76,3(61)$ \\
\hline 2. De 35 - 50 años & $22,5(19)$ \\
\hline 3. De 51 a 65 años & $1,3(1)$ \\
\hline Total & $\mathbf{1 0 0}(\mathbf{8 1})$ \\
\hline
\end{tabular}

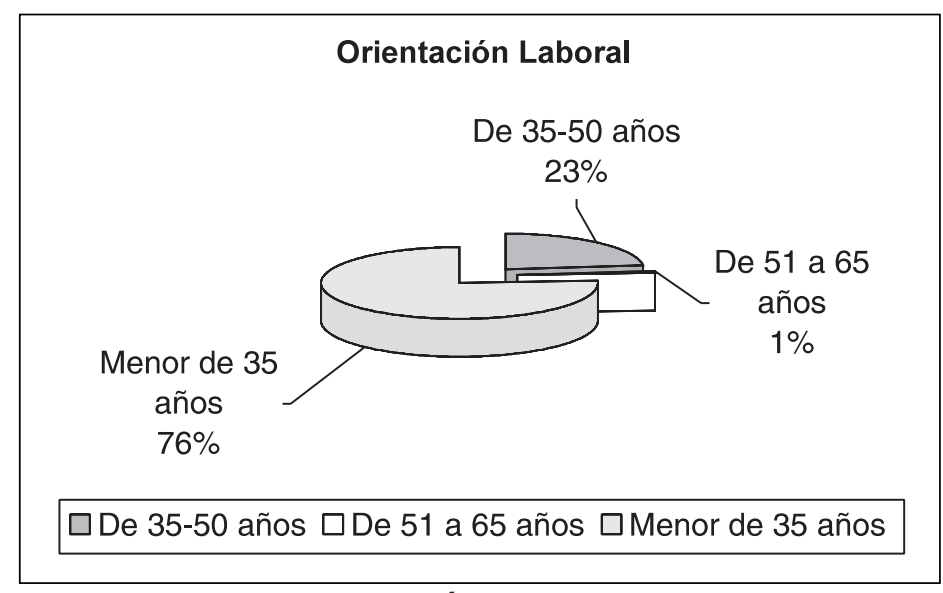

GRÁFICA 1.

Edad.

Destaca un $76 \%$ de Orientadores menor de 35 años. 
$\left.2^{\circ}\right)$ Sexo.

TABLA 2: Género.

\begin{tabular}{|c|c|}
\hline \multirow{2}{*}{ TIPOS } & NIVELES \\
\cline { 2 - 2 } & Orientación Laboral \\
\hline Varón & $27,2(22)$ \\
\hline Mujer & $72,8(59)$ \\
\hline Total & $\mathbf{1 0 0}(\mathbf{8 1})$ \\
\hline
\end{tabular}

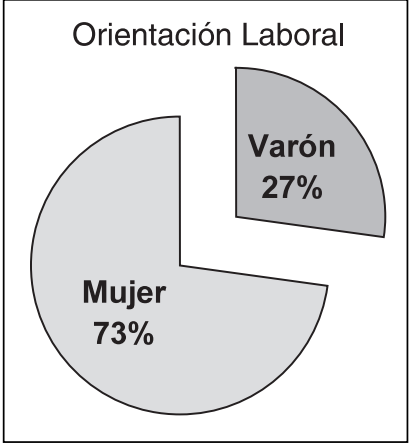

GRÁFICO 2.

Género.

En la variable género destaca el $73 \%$ de mujeres orientadoras frente a un $27 \%$ de varones.

$\left.3^{\circ}\right)$ Experiencia orientadora.

TABLA 3: Experiencia orientadora.

\begin{tabular}{|c|c|}
\hline \multirow{2}{*}{ AÑOS } & NIVELES \\
\cline { 2 - 3 } & Orientación Laboral \\
\hline Menos de 3 años & $75,3(61)$ \\
\hline De 3 a 5 años & $19,8(16)$ \\
\hline De 6 a 10 años & $3,7(3)$ \\
\hline Más de 10 años & $1,2(1)$ \\
\hline Total & $\mathbf{1 0 0}(\mathbf{8 1})$ \\
\hline
\end{tabular}

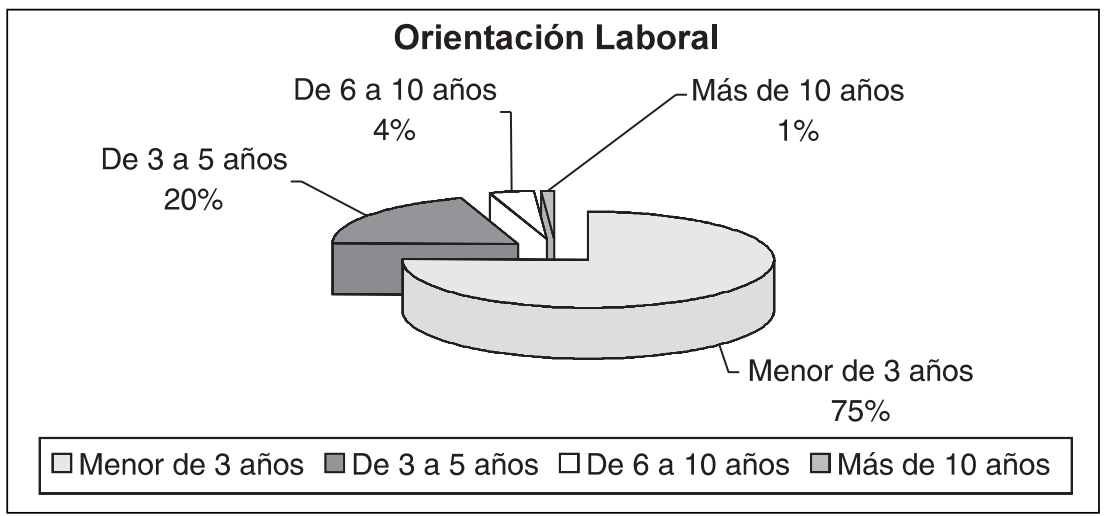

GRÁFICA 3.

Experiencia orientadora. 
Sobresale en esta variable la escasa experiencia orientadora de los profesionales de la Orientación Laboral (el 75\% es menor de 3 años).

\section{$\left.4^{\circ}\right)$ Experiencia profesional total.}

TABLA 4: Experiencia profesional general de los Orientadores.

\begin{tabular}{|c|c|}
\hline \multirow{2}{*}{ AÑOS } & NIVELES \\
\cline { 2 - 3 } & Orientación Laboral \\
\hline Menos de 3 años & $43,2(35)$ \\
\hline De 3 a 5 años & $32,1(26)$ \\
\hline De 6 a 10 años & $16(13)$ \\
\hline Más de 10 años & $8,6(7)$ \\
\hline Total & $\mathbf{9 9 , 9}(\mathbf{8 1})$ \\
\hline
\end{tabular}

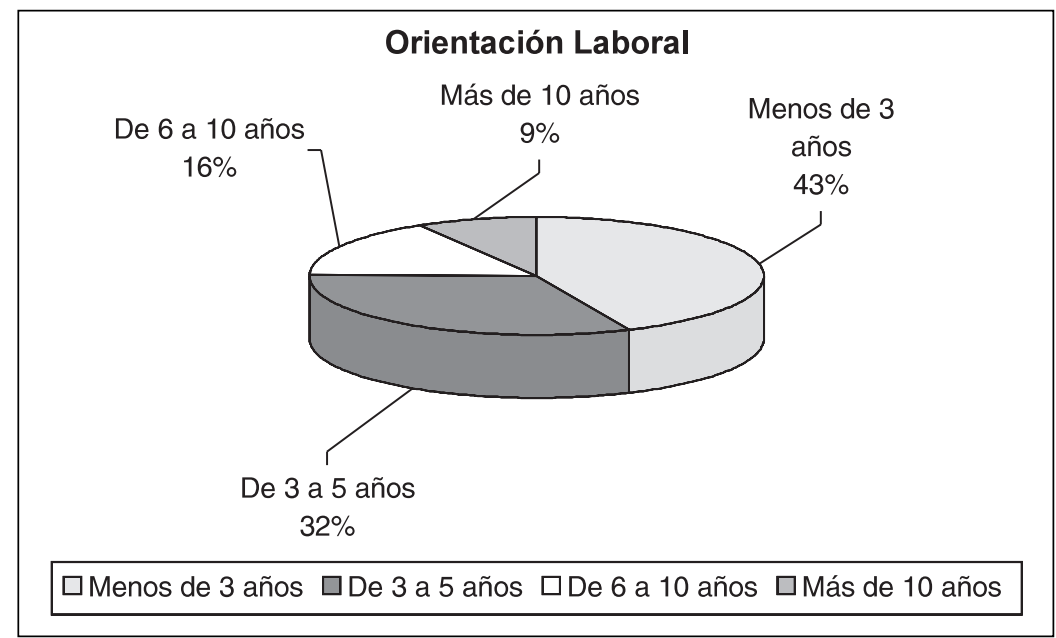

\section{GRÁFICA 4.}

Experiencia profesional total.

Destaca un $43 \%$ con menos de 3 años de experiencia profesional en general. 
$\left.5^{\circ}\right)$ Provincia.

TABLA 5: Ámbito provincial de actuación de los Orientadores.

\begin{tabular}{|l|c|}
\hline \multirow{2}{*}{ PROVINCIA } & NIVELES \\
\cline { 2 - 2 } & Orientación Laboral \\
\hline A Coruña & $35,8(29)$ \\
\hline Lugo & $14,8(12)$ \\
\hline Ourense & $11,1(9)$ \\
\hline Pontevedra & $38,3(31)$ \\
\hline Galicia & $\mathbf{1 0 0}(\mathbf{8 1})$ \\
\hline
\end{tabular}

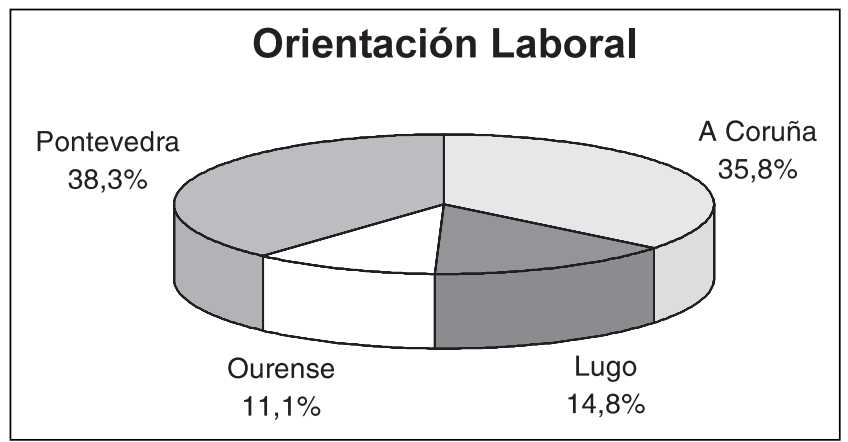

GRÁFICA 5.

Ámbito provincial de acción de los Orientadores.

Sobresale en esta variable el porcentaje alto de Orientadores Profesionales en la provincia de Pontevedra (38\%). 
$\left.6^{\circ}\right)$ Títulos académicos.

TABLA 6: Titulaciones académicas de los Orientadores.

\begin{tabular}{|l|c|}
\hline \multirow{2}{*}{$\begin{array}{c}\text { TÍTULOS } \\
\text { ACADÉMICOS }\end{array}$} & NIVELES \\
\cline { 2 - 2 } & Orientación Laboral \\
\hline Lic. Pedagogía & $28,4(23)$ \\
\hline Lic. Psicología & $22,2(18)$ \\
\hline Lic. Psicopedagogía & $12,2(10)$ \\
\hline Dipl. Educ. Social & $3,7(3)$ \\
\hline Dipl. Magisterio & $7,3(6)$ \\
\hline Dipl. Trabajo Soc. & $3,7(2)$ \\
\hline Dipl. Relac. Labor. & $12,2(10)$ \\
\hline Otras & $\mathbf{2 0 , 7}(\mathbf{1 7})$ \\
\hline
\end{tabular}

En las titulaciones académicas ocupa el primer lugar la de Pedagogía, seguida de Psicología y Psicopedagogía.

Es elevado el porcentaje del $21 \%$ de Orientadores correspondiente a otras titulaciones diferentes a las anteriores.

c) Formación académica de postgrado

TABLA 7: Modalidad de cursos de postgrado.

\begin{tabular}{|l|c|}
\hline \multicolumn{1}{|c|}{ Modalidad } & Grupo Laboral \\
\hline Especialización & 25,6 \\
\hline Máster & 54,9 \\
\hline No la tiene & 19,5 \\
\hline
\end{tabular}




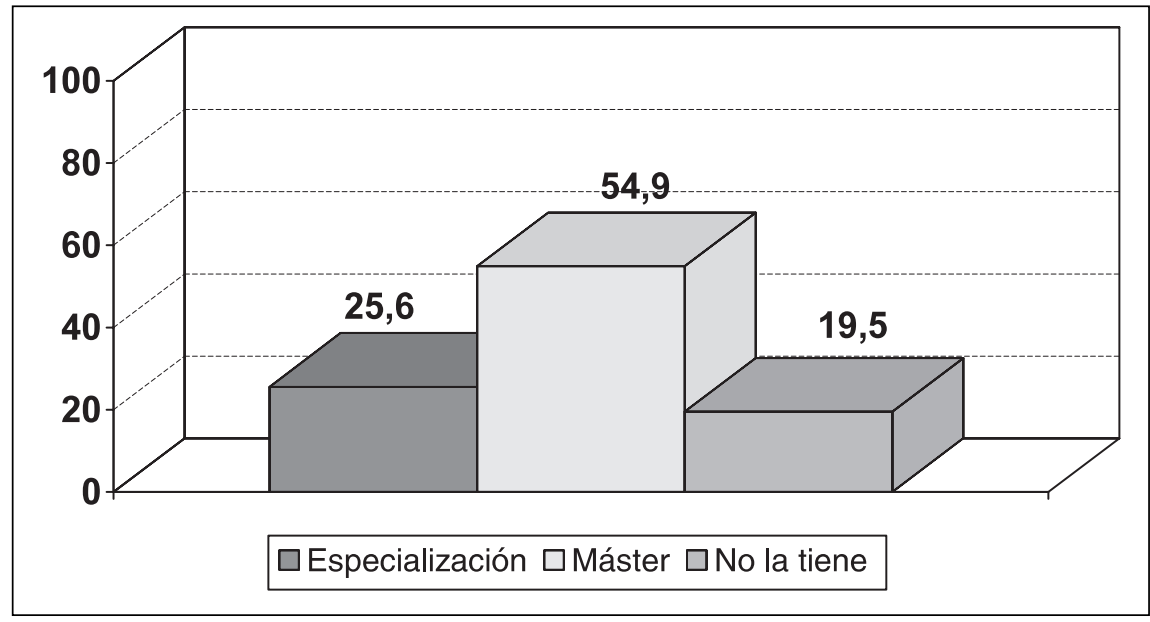

GRÁFICA 6.

Formación de postgrado.

Sobresale un elevado porcentaje de Orientadores Profesionales con titulación de Master (55\%) debido a que les favorece en su inserción laboral (existe un $60 \%$ de Oficinas Públicas de Empleo en Galicia con profesionales de Orientación que poseen este título de postgrado).

d) Sistema de acceso al puesto actual de trabajo (Orientadores Laborales)

TABLA 8: Acceso a la función orientadora.

\begin{tabular}{|l|c|}
\hline \multirow{2}{*}{ MODELO } & VALORES \\
\cline { 2 - 3 } & Porcentajes \\
\hline Concurso de méritos & 7,3 \\
\hline Contratación temporal & 82,9 \\
\hline Contratación indefinida & 3,7 \\
\hline Otros sistemas & 6,1 \\
\hline \multicolumn{1}{|c|}{ Total } & $\mathbf{1 0 0}$ \\
\hline
\end{tabular}




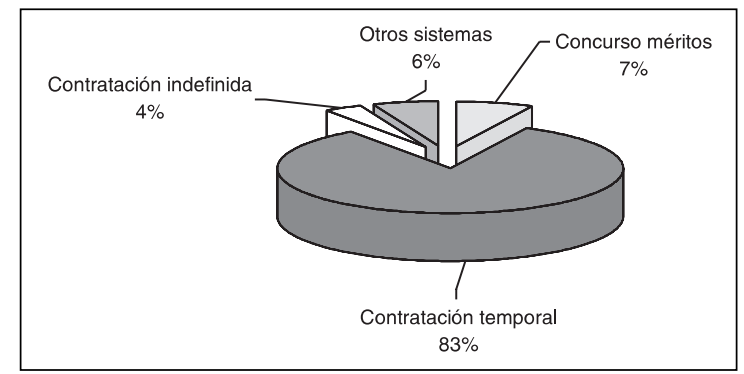

GRÁFICA 7.

Sistema de acceso al puesto de Orientador.

En los Orientadores Profesionales la contratación temporal es el modelo de acceso predominante ( $83 \%$ del colectivo encuestado). Entre otros sistemas de acceso $(6,1 \%)$ figura la contratación temporal por «obra o servicio»y el ser autónomo.

e) Servicio de Orientación en el que trabaja actualmente como Orientador Laboral

TABLA 9: Servicio Orientador en el que ejerce en la actualidad.

\begin{tabular}{|l|c|c|}
\hline \multirow{2}{*}{ MODALIDAD } & \multicolumn{2}{c|}{ VALORES } \\
\cline { 2 - 3 } & Porcentajes & Ordenación \\
\hline Oficina Pública de Empleo & 34,6 & $1^{\circ}$ \\
\hline Asociación & 14,8 & $2^{\circ}$ \\
\hline Universidad & 8,6 & $3^{\circ}$ \\
\hline Sindicato & 8,6 & $4^{\circ}$ \\
\hline Fundación & 7,4 & $5^{\circ}$ \\
\hline Empresa & 6,2 & $6^{\circ}$ \\
\hline Servicios de la Administración Laboral & 3,7 & $7^{\circ}$ \\
\hline Ayuntamiento & 3,7 & $8^{\circ}$ \\
\hline Cámara de Comercio & 3,7 & $9^{\circ}$ \\
\hline Academia TOTAL & 3,7 & $10^{\circ}$ \\
\hline Otros & 4,9 & $11^{\circ}$ \\
\hline & $\mathbf{9 9 , 9}$ & \\
\hline
\end{tabular}




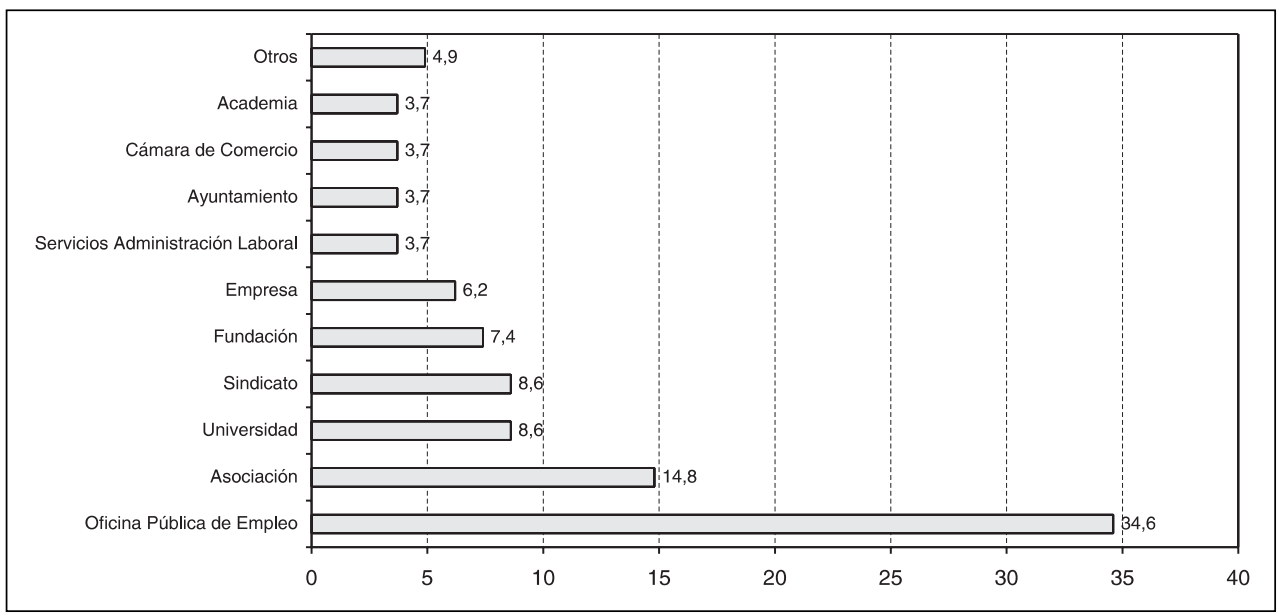

GRÁFICA 8.

Servicio de Orientación en el que trabaja actualmente.

En esta variable destaca el alto porcentaje (35\%) de Orientadores Laborales que trabajan en Oficinas Públicas de Empleo.

En el apartado «otros» figura: Fondo de Formación del Empleo, Diputación Provincial y Centros para el desarrollo comarcal.

\section{Elaboración y aplicación del Cuestionario de competencias orientadoras}

Este instrumento consta de tres partes, la primera (ya expuesta) se refiere a datos personales y profesionales de los Orientadores y la segunda trata de las competencias de orientación y consta de 75 items con las dimensiones siguientes:

$1^{\text {a }}$. Competencias generales ( 9 items).

$2^{\mathrm{a}}$. Diagnóstico (6 cuestiones).

$3^{\mathrm{a}}$. Orientación Personal y Grupal (10 items).

$4^{\mathrm{a}}$. Desarrollo personal (5 cuestiones).

$5^{\mathrm{a}}$. Asesoramiento (8 items).

6. Gestión de la información (7 items).

$7^{\mathrm{a}}$. Consulta y Coordinación (5 items).

8 . Investigación y Evaluación (7 cuestiones).

9a. Dirección de Servicios y/o Programas Orientadores (7 items).

$10^{\mathrm{a}}$. Relaciones con la Comunidad Social (6 items).

$11^{\mathrm{a}}$. Inserción Profesional (5 cuestiones). 
La tercera se refiere a las dimensiones de la Orientación específicas citadas anteriormente.

Consta la segunda parte de items de respuesta cerrada que van de 0-5, a cumplimentar en tres columnas relativas a la frecuencia de realización de competencias orientadoras, a la frecuencia con que éstas se deberían efectuar y a la preparación actual en su dominio por los Orientadores Profesionales.

La valoración de los items de respuesta cerrada abarca entre 0 (ninguna) a 5 (mucha).

Los cuestionarios se redactaron y aplicaron en gallego para facilitar la respuesta de los Orientadores todos ellos conocedores de la Lengua Gallega.

\section{Estudio estadístico de los datos obtenidos del cuestionario de competencias de Orientación}

\section{Fiabilidad y validez}

\section{Fiabilidad}

El cálculo de la fiabilidad mediante el instrumento Alfa de Cronbach, referido a las tres columnas citadas (competencias reales, ideales y con preparación actual) y al grupo de Orientadores Laborales destaca el elevado coeficiente de los datos obtenidos (todos superiores a 0,90 ), lo que representa una elevada consistencia interna.

\section{TABLA 10: Análisis del Cuestionario de Competencias Orientadoras.}

\begin{tabular}{|l|c|}
\hline \multicolumn{1}{|c|}{ Columnas } & Grupo Orientación Laboral \\
\hline A (competencias realizadas) & 0,9084 \\
\hline B (competencias idealizadas) & 0,9183 \\
\hline C (competencias con preparación) & 0,9478 \\
\hline
\end{tabular}

Validez

Se interpreta la validez de un instrumento investigador como la cualidad por la que se evalúa lo que se pretende hacer y por ello es preciso explicitar el propósito principal el contenido de la prueba en cuestión.

El instrumento de recogida de datos utilizado es un cuestionario de competencias orientadoras que se propone recabar información, respecto a la valoración que los Orientadores Profesionales conceden a las competencias que realizan en su función, a las que deberían efectuar y a su preparación actual en el dominio de dichas competencias.

Asimismo se pretende la valoración de las dimensiones orientadoras que agrupan a los items respectivos, ordenándolas según su importancia.

La primera parte del instrumento se refiere a datos de identificación personal y profesional de los Orientadores Laborales. 
Para saber si se pueden lograr los objetivos propuestos se necesita probar la validez desde la perspectiva del contenido básicamente, puesto que al ser un instrumento de opinión carece de sentido referirse a su predicción.

Interpretamos que un instrumento de valoración posee validez de contenido si los items que se incluyen en la prueba son representativos y suficientes en cuanto al conjunto de las competencias que constituyen el ámbito que se va a evaluar.

Para estimar la validez de contenido, Brow propone desarrollar un instrumento de clasificación para valorar la calidad de la prueba en varias dimensiones relevantes como son la aptitud del contenido, la amplitud de las habilidades (en nuestro caso competencias) y lo apropiado del formato.

En nuestra investigación se aplicó una prueba de validación que permitía valorar en un escala de 1 a 10 la pertinencia de cada item del Cuestionario de competencias orientadoras a aplicar y una escala final que se refería a los aspectos siguientes del cuestionario citado en su globalidad:

- Presentación.

- Amplitud de la prueba.

- Claridad de la redacción.

- Pertinencia de las dimensiones del instrumento.

- Concreción de los items.

El instrumento de validación se aplicó a un grupo de doce expertos (profesores universitarios de Orientación Educativa y Profesional y Orientadores especialistas de reconocido prestigio profesional). Para ello se siguió la técnica Delphi con feed-back informativo.

Para facilitar la interpretación de los resultados se recodificaron las valoraciones de 1-10 en la escala siguiente de calificación:

$$
\begin{aligned}
& \text { 9-10: Muy bien } \\
& 7 \text {-8: Bien } \\
& \text { 5-6: Regular } \\
& \text { 3-4: Insuficiente } \\
& \text { 1-3: Mal }
\end{aligned}
$$

Obteniendo el promedio que cada experto concedió a cada una de las dimensiones de las competencias orientadoras que aparecen en las columnas A (frecuencia de la realización actual de las competencias), B (deber de efectuarlas) y C (preparación actual en su dominio) se extrajeron los resultados que siguen: 
TABLA 11: Promedio de valoración de las dimensiones orientadoras.

\begin{tabular}{|l|c|c|c|}
\hline \multirow{2}{*}{\begin{tabular}{c}
\multirow{2}{*}{$\begin{array}{c}\text { DIMENSIONES } \\
\text { ORIENTADORAS }\end{array}$} \\
\cline { 2 - 4 }
\end{tabular}} & $\begin{array}{c}\text { Frecuencia } \\
\text { realización actual }\end{array}$ & $\begin{array}{c}\text { Deber de efectuar } \\
\text { las competencias }\end{array}$ & $\begin{array}{c}\text { Preparación actual } \\
\text { competencias }\end{array}$ \\
\hline Competencias generales & 9,13 & 9,13 & 8,86 \\
\hline Diagnóstico & 8,95 & 9,33 & 9,08 \\
\hline $\begin{array}{l}\text { Orientación Profesional y } \\
\text { Grupal }\end{array}$ & 9,15 & 9,02 & 9,22 \\
\hline Desarrollo Profesional & 8,90 & 9,00 & 9,05 \\
\hline Asesoramiento & 9,37 & 9,37 & 9,37 \\
\hline $\begin{array}{l}\text { Gestión de la Comprobación } \\
\text { y recursos }\end{array}$ & 8,75 & 8,42 & 8,71 \\
\hline Consulta y Coordinación & 8,60 & 8,65 & 8,31 \\
\hline Comprobación y Evaluación & 9,03 & 8,85 & 9,10 \\
\hline $\begin{array}{l}\text { Dirección de Servicios y/o } \\
\text { programas orientadores }\end{array}$ & 9,00 & 9,03 & 9,03 \\
\hline $\begin{array}{l}\text { Relaciones con la Comunidad } \\
\text { Social }\end{array}$ & 8,91 & 8,91 & 8,92 \\
\hline Comprobación Profesional & 9,40 & 9,15 & 9,20 \\
\hline
\end{tabular}

En lo concerniente a la validez del cuestionario de competencias orientadoras la basamos en la de contenido por ser la apropiada en esta modalidad de instrumento y podemos observar una elevada valoración de cada una de las dimensiones que corresponden a todos los items de la prueba.

En la tabla 11 figuran los promedios correspondientes a la valoración de la frecuencia de realización de competencias, deber de efectuarlas y preparación actual en su dominio observándose que son bastante altos.

Respecto a la opinión global del instrumento aplicado, además de los resultados estadísticos obtenidos en la fiabilidad del mismo (todos los valores logrados del coeficiente Alpha de Cronbach superan el 0,90 ) los juicios observados conceden una suficiente validez para aplicar el cuestionario referido.

Por todo lo expuesto creemos que la prueba efectuada reúne los requisitos e indicadores que se pueden exigir a un instrumento de evaluación de esta modalidad.

Asimismo su lectura atenta posibilita una reflexión detenida de la actividad efectuada por los Orientadores, por lo que su utilización nos parece acertada en cuanto a los propósitos de la investigación propuesta. 
Análisis de varianza para la comprobación de posibles diferencias significativas

a) Comprobación de los promedios de edad y realización de competencias de los Orientadores (Columna A).

TABLA 12: Análisis de varianza entre edad y realización de competencias de Orientación.

\begin{tabular}{|c|c|c|c|c|c|}
\hline $\begin{array}{c}\text { Fuentes de } \\
\text { Variación (F.V.) }\end{array}$ & $\begin{array}{c}\text { Suma de } \\
\text { Cuadrados } \\
\text { (S.C.) }\end{array}$ & $\begin{array}{c}\text { Grados de } \\
\text { Libertad } \\
\text { (G.L.) }\end{array}$ & $\begin{array}{c}\text { Medias } \\
\text { Cuadráticas } \\
\text { (M.C.) }\end{array}$ & $\mathbf{F}$ & Significación \\
\hline Entre grupos & 44714,866 & 2 & 22357,433 & \multirow{3}{*}{8,761} & \multirow{3}{*}{0,001} \\
\hline Dentro de grupos & 579266,878 & 227 & 2551,836 & & \\
\hline TOTAL & 623981,744 & 229 & - & & \\
\hline
\end{tabular}

Dado el valor tabular de $\mathrm{F}=8,761$ al nivel de significación de 0,05 y con significación 0,001 (2 y 227 G.L.), revela que hay diferencias significativas al ser $0,001<0,05$.

b) Comprobación de los promedios de edad y deber de realizar las competencias de los Orientadores (Columna B).

TABLA 13: Análisis de varianza entre edad y deber de realizar las competencias orientadoras.

\begin{tabular}{|c|c|c|c|c|c|}
\hline $\begin{array}{c}\text { Fuentes de } \\
\text { Variación (F.V.) }\end{array}$ & $\begin{array}{c}\text { Suma de } \\
\text { Cuadrados } \\
\text { (S.C.) }\end{array}$ & $\begin{array}{c}\text { Grados de } \\
\text { Libertad } \\
\text { (G.L.) }\end{array}$ & $\begin{array}{c}\text { Medias } \\
\text { Cuadráticas } \\
\text { (M.C.) }\end{array}$ & F & Significación \\
\hline Entre grupos & 32804,329 & 2 & 16402,164 & \multirow{2}{*}{8,9} & \multirow{2}{*}{0,001} \\
\hline Dentro de grupos & 422027,667 & 229 & 1842,916 & \\
\cline { 1 - 5 } TOTAL & $\mathbf{4 5 4 8 3 1 , 9 9 6}$ & $\mathbf{2 3 1}$ & - & \\
\hline
\end{tabular}

Dado el valor tabular de $\mathrm{F}=8,9$ al nivel de significación de 0,05 (2 y 229 G.L.) y con significación 0,001 , revela que hay diferencias significativas al ser 0,001<0,05.

c) Comprobación de los promedios de edad y preparación actual en las competencias de los Orientadores (Columna C). 
TABLA 14: Análisis de varianza entre edad y preparación en las competencias orientadoras.

\begin{tabular}{|l|c|c|c|c|c|}
\hline $\begin{array}{c}\text { Fuentes de } \\
\text { Variación (F.V.) }\end{array}$ & $\begin{array}{c}\text { Suma de } \\
\text { Cuadrados } \\
\text { (S.C.) }\end{array}$ & $\begin{array}{c}\text { Grados de } \\
\text { Libertad } \\
\text { (G.L.) }\end{array}$ & $\begin{array}{c}\text { Medias } \\
\text { Cuadráticas } \\
\text { (M.C.) }\end{array}$ & F & Significación \\
\hline Entre grupos & 43308,955 & 2 & 21654,477 & \multirow{2}{*}{7,717} & \multirow{2}{*}{0,001} \\
\cline { 1 - 4 } Dentro de grupos & 642619,834 & 229 & 2806,200 & \\
\cline { 1 - 4 } TOTAL & $\mathbf{6 8 5 9 2 8 , 7 8 9}$ & $\mathbf{2 3 1}$ & - & \\
\hline
\end{tabular}

Dado el valor tabular de $\mathrm{F}=7,717$ al nivel de significación de 0,05 (2 y 229 G.L.) y con significación 0,001, expresa que existen diferencias significativas al ser $0,001<0,05$.

d) Comprobación de los promedios de sexo, y realización de competencias de los Orientadores (Columna A).

TABLA 15: Análisis de varianza entre sexo y realización de competencias.

\begin{tabular}{|c|c|c|c|c|c|}
\hline $\begin{array}{c}\text { Fuentes de } \\
\text { Variación (F.V.) }\end{array}$ & $\begin{array}{c}\text { Suma de } \\
\text { Cuadrados } \\
\text { (S.C.) }\end{array}$ & $\begin{array}{c}\text { Grados de } \\
\text { Libertad } \\
\text { (G.L.) }\end{array}$ & $\begin{array}{c}\text { Medias } \\
\text { Cuadráticas } \\
\text { (M.C.) }\end{array}$ & F & Significación \\
\hline Entre grupos & 3019,948 & 1 & 3019,948 & \multirow{2}{*}{1,037} & \multirow{2}{*}{0,310} \\
\cline { 1 - 4 } Dentro de grupos & 666869,489 & 229 & 2912,094 & \\
\cline { 1 - 4 } TOTAL & $\mathbf{6 6 9 8 8 9 , 4 3 7}$ & $\mathbf{2 3 0}$ & & \\
\hline
\end{tabular}

Dado el valor tabular de $\mathrm{F}=1,037$ al nivel de significación de 0,05 (1 y 229 G.L.) y con significación 0,310 , expresa que no existen diferencias significativas al ser $0,310>0,05$.

e) Comprobación de los promedios de sexo y competencias que deberían darse en los Orientadores (Columna B).

TABLA 16: Análisis de varianza entre sexo y deber de realizar las competencias.

\begin{tabular}{|l|c|c|c|c|c|}
\hline $\begin{array}{c}\text { Fuentes de } \\
\text { Variación (F.V.) }\end{array}$ & $\begin{array}{c}\text { Suma de } \\
\text { Cuadrados } \\
\text { (S.C.) }\end{array}$ & $\begin{array}{c}\text { Grados de } \\
\text { Libertad } \\
\text { (G.L.) }\end{array}$ & $\begin{array}{c}\text { Medias } \\
\text { Cuadráticas } \\
\text { (M.C.) }\end{array}$ & F & Significación \\
\hline Entre grupos & 18735,818 & 1 & 18735,818 & \multirow{2}{*}{9,902} & \multirow{2}{*}{0,002} \\
\cline { 1 - 4 } Dentro de grupos & 437083,899 & 231 & 1892,138 & \\
\cline { 1 - 4 } TOTAL & $\mathbf{4 5 5 8 1 9 , 7 1 7}$ & $\mathbf{2 3 2}$ & & \\
\hline
\end{tabular}


Dado el valor tabular de $\mathrm{F}=9,902$ al nivel de significación de 0,05 (1 y 231 G.L.) y con significación 0,002 , indica que existen diferencias significativas al ser $0,002<0,05$.

\section{Escalamiento multidimensional de las competencias orientadoras}

La frecuencia de realización de las dimensiones orientadoras según promedios y ordenación es la siguiente:

$1^{\circ}$. Asesoramiento $(2,93)$

$2^{\circ}$. Orientación $(3,06)$

$3^{\circ}$. Diagnóstico $(3,58)$

$4^{\circ}$. Inserción Profesional (5)

$5^{\circ}$. Desarrollo Profesional $(5,24)$

$6^{\circ}$. Gestión de la Información $(5,32)$

$7^{\circ}$. Consulta y Coordinación $(6,69)$

$8^{\circ}$. Relaciones con la Comunidad $(7,33)$

$9^{\circ}$. Dirección de los Servicios y/o Programas $(7,68)$

$10^{\circ}$. Investigación y Evaluación $(8,39)$

Las tres primeras dimensiones orientadoras son el Asesoramiento, el Diagnóstico y la Orientación y las tres últimas la Investigación y Evaluación, la Dirección de Servicios y/o Programas y las Relaciones con la Comunidad.

Las dimensiones de Diagnóstico, Asesoramiento y Orientación (Personal y Grupal) son las preferidas por los Orientadores Laborales y las que consideran más importantes. Expresan las que se realizan por todos los Orientadores con más frecuencia.

Las dimensiones de Desarrollo Profesional, Gestión de la Información y recursos e Inserción Profesional son relevantes para los Orientadores Laborales, que valoran menos las de Relaciones con la Comunidad, la Dirección de Servicios y/o Programas de Orientación y la Investigación y Evaluación.

En general el escalamiento percibido se considera normal en relación con la frecuencia de realización de competencias profesionales por los Orientadores Laborales.

\section{A modo de conclusión}

El perfil dominante del Orientador Laboral que ejerce en la Comunidad Autónoma de Galicia es una persona joven, de sexo femenino, con reducida experiencia profesional en general y orientadora específicamente, con titulación académica de Licenciatura en Pedagogía, con acceso por contratación temporal y que ejerce en una Oficina Pública de Empleo.

Como competencias profesionales de los Orientadores sobresalen las referentes a las conductas éticas y actitudinales de respeto e interés personal por los orientandos. 
Como competencias de los Orientadores Laborales sobresalen las de Asesoramiento, Orientación Profesional y Diagnóstico.

\section{Referencias bibliográficas}

Anta, G. (1998): Procesos de acreditación y certificación de la competencia laboral. Madrid: O.E.I.

Ballesteros, B. (1996): Análisis de las competencias del orientador y sus implicaciones: un estudio en siete países de la Unión Europea. Tesis Doctoral. Madrid: UNED (Inédita).

Blagg, N. y otros (1993): Development of transferible skills in learners. Sheffield: Employment Departament.

Brotherton, S. (1996): Counselor education for the twenty-first century. Westport: Bergin.

Bunk, G. P. (1994): «La transmisión de competencias de la formación y perfeccionamiento profesionales». Revista Europea de Formación Profesional, 1, 8-14.

CERI (1996): Choisir son avenir. París: OCDE.

Colardyn, D. (1996): La gestion des competences. Perspectives internacionales. París: P.U.F.

Drummond, R. (2000): Appraisal procedures for Counselor and helping profesionnals. Columbus, Ohio: Merrill ( $4^{\mathrm{a}}$ ed.).

Engels, D. W. y Dameron, F. J. (1990): The professional counselor. Competencies, performance and assessment. Alexandria, VA: Guidelines.

Eraut, M. (1997): Developing profesional knowledge and competence. Londres: The Falmer Press.

Hiebert, B. (2000a): «Competencies for providing quality career services: a look at Canadian standars for career development». Revista Espaola de Orientación y Psicopedagogía, vol. 11, 19, 519.

Hiebert, B. (2000b): «Practioner competencies: changing the roles in the new millennium». IAEGV (Internacional Conference for Vocational Guidance). Berlín.

Hodkinson, P. e Issit, M. (1995): The challenge of competence: professionalism through vocational education and training. Londres: Cassell.

ISFOL (1992): Competenze transversali e comportamento organizzativo. La abilitá di base per il lavoro che cambia. Roma: Instituto per lo Sviluppo della Formazione Professionale dei Lavoratori.

Joras, M. (1995): Le bilan de compétences. París: P.U.F.

Joras, M. y Ravier, J. (1993): Compendre le bilan de compétences. París: Edit. Loaisons.

Kottler, J.A. (1997): Finding your way as a counselor. Alexandria, VA: A.C.A.

Landeta, J. (1999): El método Delphi. Barcelona: Ariel.

Le Boterf, G. (1994): De la competence. París: Les Editions d'Organisations.

Lee, C. y Walz, G. (1998): Social action. A mandate for counselor. Alexnadria, VA: A.C.A.

Levy-Leboyer, C. (1996): La gestion des competences. París: Editions D’Organisation. (Traducción española, 1997). La gestión de las competencias. Barcelona: Ediciones Gestión 2000).

Levy-Leboyer, C. (1997): La gestión de las competencias. Barcelona: Ediciones Gestión 2000.

Mazariegos, A. y otras (1999): Competencias transversales. Un reto para la formación profesional. Barcelona: FORCEM.

Mertens, L. (1998): La gestión por competencia laboral en la empresa y la formación profesional. Madrid: O.I.T.

Ministerio de Educación y Cultura (1998): Nuevo programa nacional de Formación Profesional. Madrid: Secretaría general del Consejo General de Formación Profesional. 
Nijhof, W. y Streumer, J. (1998): Key qualifications in work and education. Dordracht: Kluwet Academic Pub.

Repetto, E. (2000): «Qualification standards for career services provides. Qualifications standards for educational and vocational guidance services self». International Conference AIOSP. Berlín.

Rodríguez Moreno, Ma. L. (1992): El mundo del trabajo y las funciones del Orientador. Barcelona: Barcanova.

RVQ (1986): Review of Vocational Qualifications: Final Report. Londres: HMSO.

Sanz Oro, R. (1999): Los Departamentos de Orientación en Educación Secundaria: Roles y funciones. Barcelona: Editorial Cedecs S.L.

Sanz, R. y Sobrado, L. (1998): «Roles y funciones de los Orientadores». Revista de Investigación Educativa, Vol. 16, 2, 25-57.

Sobrado, L. (1997): «Evaluación de las competencias profesionales de los Orientadores Escolares». Revista de Investigación Educativa, Vol. 15, 1, 83-102.

Sobrado, L. (2002): Diagnóstico en Educación. Madrid: Biblioteca Nueva.

Sobrado, L. y Ocampo, C. (2001): Evaluación Psicopedagógica y Orientación Educativa (3ª edición). Barcelona: Estel / Servicio de Publicaciones de la Universidad.

Watts, A.G. y colaboradores (1992): Perfiles profesionales de los consejeros de Orientación en la Comunidad Europea. Informe de Síntesis. Berlín: CEDEFOP.

Wolf, A. (1995): Competence based assessment. Buckingham: Open University Press.

Fecha de recepción: 08-01-03

Fecha de revisión: 28-09-03

Fecha de aceptación: 27-10-03 